Research Article

\title{
Strong Convergence Theorems for a Pair of Strictly Pseudononspreading Mappings
}

\author{
Bin-Chao Deng and Tong Chen \\ School of Management, Tianjin University, Tianjin 300072, China \\ Correspondence should be addressed to Bin-Chao Deng; dbchao1985@tju.edu.cn \\ Received 18 December 2012; Accepted 17 June 2013 \\ Academic Editor: Krassimir T. Atanassov
}

Copyright (C) 2013 B.-C. Deng and T. Chen. This is an open access article distributed under the Creative Commons Attribution License, which permits unrestricted use, distribution, and reproduction in any medium, provided the original work is properly cited.

Let $H$ be a real Hilbert space. Let $T_{1}, T_{2}: H \rightarrow H$ be $k_{1}-, k_{2}$-strictly pseudononspreading mappings; let $\left\{\alpha_{n}\right\}$ and $\left\{\beta_{n}\right\}$ be two real sequences in $(0,1)$. For given $x_{0} \in H$, the sequence $\left\{x_{n}\right\}$ is generated iteratively by $x_{n+1}=\beta_{n} x_{n}+\left(1-\beta_{n}\right) T_{w_{1}}\left(\alpha_{n} \gamma f\left(x_{n}\right)+(I-\right.$ $\left.\left.\mu \alpha_{n} B\right) T_{w_{2}} x_{n}\right), \forall n \in N$, where $T_{w_{i}}=\left(1-w_{i}\right) I+w_{i} T_{i}$ with $i=1,2$ and $B: H \rightarrow H$ is strongly monotone and Lipschitzian. Under some mild conditions on parameters $\left\{\alpha_{n}\right\}$ and $\left\{\beta_{n}\right\}$, we prove that the sequence $\left\{x_{n}\right\}$ converges strongly to the set $F_{i x}\left(T_{1}\right) \cap F_{i x}\left(T_{2}\right)$ of fixed points of a pair of strictly pseudononspreading mappings $T_{1}$ and $T_{2}$.

\section{Introduction}

Let $H$ be a real Hilbert space whose inner product and norm are denoted by $\langle\cdot, \cdot\rangle$ and $\|\cdot\|$, and let $C$ be a nonempty, closed, and convex subset of $H$, respectively. Recall the following definitions.

Definition 1. Let $A: C \rightarrow H$ be a nonlinear mapping.

(1) $A$ is said to be monotone if

$$
\langle A x-A y, x-y\rangle \geq 0, \quad \forall x, y \in C .
$$

(2) $A$ is said to be strongly monotone if there exists a constant $\alpha>0$ such that

$$
\langle A x-A y, x-y\rangle \geq \alpha\|x-y\|^{2}, \quad \forall x, y \in C .
$$

For such a case, $A$ is said to be $\alpha$-strongly-monotone.

(3) $A$ is said to be inverse strongly if there exists a constant $\alpha>0$ such that

$$
\langle A x-A y, x-y\rangle \geq \alpha\|A x-A y\|^{2}, \quad \forall x, y \in C .
$$

For such a case, $A$ is said to be $\alpha$-inverse-strongly monotone.
The classical variational inequality which is denoted by $\mathrm{VI}(A, C)$ is to find $x \in C$ such that

$$
\langle A x, y-x\rangle \geq 0, \quad \forall y \in C .
$$

The variational inequality has been extensively studied in the literature; see, for example, $[1,2]$ and the reference therein. Recall that $T$ is a nonexpansive mapping of $C$ into itself; that is,

$$
\|T x-T y\| \leq\|x-y\|, \quad \forall x, y \in C .
$$

The set of fixed points of $T$ is the set $F_{i x}(T)=\{x \in H, T x=$ $x\}$.

In 2011, Osilike and Isiogugu [3] introduced a new class of mappings, the so-called $k$-strictly pseudononspreading; that is, a mapping $T: D(T) \subseteq H \rightarrow H$ is $k$-strictly pseudononspreading if there exists $k \in[0,1)$ such that

$$
\begin{aligned}
\|T x-T y\|^{2} \leq & \|x-y\|^{2}+k\|x-T x-(y-T y)\|^{2} \\
& +2\langle x-T x, y-T y\rangle,
\end{aligned}
$$

for all $x, y \in D(T)$. They showed that the class of nonspreading mappings is properly contained in the class of strictly pseudononspreading mappings. 
The iteration procedure of Mann's type for approximating fixed points of a nonexpansive mapping $T$ is the following: $x_{1} \in C$ and

$$
x_{n+1}=\alpha_{n} x_{n}+\left(1-\alpha_{n}\right) T x_{n}, \quad \forall n \in N
$$

where $\left\{\alpha_{n}\right\}$ is a sequence in $[0,1]$; see [4]. For two nonexpansive mappings $S$ and $T$, Takahashi and Tamura [5] considered the following iteration procedure: $x_{1} \in C$ and

$$
\begin{array}{r}
x_{n+1}=\alpha_{n} x_{n}+\left(1-\alpha_{n}\right) S\left(\beta_{n} x_{n}+\left(1-\beta_{n}\right) T x_{n}\right), \\
\forall n \in N,
\end{array}
$$

where $\left\{\alpha_{n}\right\}$ and $\left\{\beta_{n}\right\}$ are sequences in $[0,1]$.

In 2010, Tian [6] introduced the following general viscosity iterative scheme for finding an element of set of solutions to the fixed point of nonexpansive mapping in Hilbert space. Define sequence $\left\{x_{n}\right\}$ by

$$
x_{n+1}=\alpha_{n} \gamma f\left(x_{n}\right)+\left(I-\mu \alpha_{n} B\right) T x_{n}, \quad n \geq 0,
$$

where $B$ is $k$-Lipschitzian and $\eta$-strongly monotone operator and $T$ is a nonexpansive mapping on $H$; then he proved that if the sequence $\left\{\alpha_{n}\right\}$ satisfies appropriate conditions, the sequence $\left\{x_{n}\right\}$ generated by (9) converges strongly to the unique solution $x^{*} \in C$ of the variational inequality

$$
\left\langle(\gamma f-\mu B) x^{*}, x-x^{*}\right\rangle \leq 0, \quad \forall x \in C
$$

where $C=\operatorname{Fix}(T)$.

In this paper, motivated by Takahashi and Tamura [5] and Tian [6], we introduce the following iteration scheme for finding a common point of the set $F_{i x}\left(T_{1}\right) \bigcap F_{i x}\left(T_{2}\right)$ of fixed points of a pair of strictly pseudononspreading mappings $T_{1}$ and $T_{2}$ :

$$
\begin{aligned}
x_{n+1}= & \beta_{n} x_{n}+\left(1-\beta_{n}\right) T_{\omega_{1}} \\
& \times\left(\alpha_{n} \gamma f\left(x_{n}\right)\right. \\
& \left.\quad+\left(I-\mu \alpha_{n} B\right) T_{\omega_{2}} x_{n}\right), \quad \forall n \in N,
\end{aligned}
$$

where $T_{\omega_{i}}=\left(1-\omega_{i}\right) I+\omega_{i} T_{i}$ with $i=1,2$ and $B$ : $H \rightarrow H$ is $\eta$-strongly monotone and $k$-Lipschitzian on $H$ with $k>0, \eta>0$. Under suitable conditions, we prove a strong convergence theorem, which is different from the results of general viscosity iterative scheme in [6].

\section{Preliminaries}

We need some facts and tools in Hilbert space $H$ which are listed as in the following lemmas.

Definition 2. A mapping $T$ is said to be demiclosed, if for any sequence $\left\{x_{n}\right\}$ which weakly converges to $y$, and if the sequence $\left\{T x_{n}\right\}$ strongly converges to $z$, then $T(y)=z$.
Definition 3. $T: H \rightarrow H$ is called demicontractive on $H$, if there exists a constant $\alpha<1$ such that

$$
\begin{array}{r}
\|T x-q\|^{2} \leq\|x-q\|^{2}+\alpha\|x-T x\|^{2}, \\
\forall(x, q) \in H \times F_{i x}(T) .
\end{array}
$$

Remark 4. Every $k$-strictly pseudononspreading mapping with a nonempty fixed point set $F_{i x}(T)$ is demicontractive (see $[7,8])$.

Remark 5 (see [9]). Let $T$ be a $\alpha$-demicontractive mapping on $H$ with $F_{i x}(T) \neq \emptyset$ and $T_{\omega}=(1-\omega) I+\omega T$ for $\omega \in(0, \infty)$.

(A1) $T \alpha$-demicontractive is equivalent to

$$
\begin{aligned}
\langle x-T x, x-q\rangle & \geq \frac{1}{2}(1-\alpha)\|x-T x\|^{2}, \\
& \forall(x, q) \in H \times F_{i x}(T) .
\end{aligned}
$$

(A2) $F_{i x}(T)=F_{i x}\left(T_{\omega}\right)$ if $\omega \neq 0$.

Remark 6. According to $I-T_{\omega}=\omega(I-T)$ with $T$ being a $k$-strictly pseudononspreading mapping, we obtain

$$
\begin{aligned}
\left\langle x-T_{\omega} x, x-q\right\rangle \geq & \frac{\omega(1-k)}{2}\|x-T x\|^{2}, \\
& \forall(x, q) \in H \times F_{i x}(T) .
\end{aligned}
$$

Proposition 7 (see [3]). Let $C$ be a nonempty closed convex subset of a real Hilbert space $H$, and let $T: C \rightarrow C$ be a $k$ strictly pseudononspreading mapping. If $F_{i x}(T) \neq \emptyset$, then it is closed and convex.

Proposition 8 (see [3]). Let $C$ be a nonempty closed convex subset of a real Hilbert space $H$, and let $T: C \rightarrow C$ be a k-strictly pseudononspreading mapping. Then $(I-T)$ is demiclosed at 0 .

Lemma 9. Let $H$ be a real Hilbert space. The following expressions hold:

(i) $\|t x+(1-t) y\|^{2}=t\|x\|^{2}+(1-t)\|y\|^{2}-t(1-t)\|x-y\|^{2}$, $\forall x, y \in H, \forall t \in[0,1]$.

(ii) $\|x+y\|^{2} \leq\|x\|^{2}+2\langle y, x+y\rangle, \forall x, y \in H$.

Lemma 10 (see [10]). Let $C$ be a closed convex subset of $a$ Hilbert space $H$, and let $T: H \rightarrow H$ be a $k$-strictly pseudononspreading mapping with a nonempty fixed point set. Let $k \leq \omega<1$ be fixed, and define $T_{\omega}: C \rightarrow C$ by

$$
T_{\omega} x=(1-\omega) x+\omega T x, \quad \forall x \in C .
$$

Then $F_{i x}\left(T_{\omega}\right)=F_{i x}(T)$.

Lemma 11 (see [11]). Assume $\left\{\alpha_{n}\right\}$ is a sequence of nonnegative real numbers such that

$$
\alpha_{n+1} \leq\left(1-\gamma_{n}\right) \alpha_{n}+\delta_{n}, \quad n \geq 0,
$$


where $\left\{\gamma_{n}\right\}$ is a sequence in $(0,1)$ and $\left\{\delta_{n}\right\}$ is a sequence in $\mathbb{R}$ such that

(i) $\sum_{n=1}^{\infty} \gamma_{n}=\infty$

(ii) $\lim \sup _{n \rightarrow \infty}\left(\delta_{n} / \gamma_{n}\right)=0$ or $\sum_{n=1}^{\infty}\left|\delta_{n}\right|<\infty$.

Then $\lim _{n \rightarrow \infty} \alpha_{n}=0$.

Lemma 12 (see [12]). Let $\left\{\mathscr{T}_{n}\right\}$ be a sequence of real numbers that does not decrease at infinity, in the sense that there exists a subsequence $\left\{\mathscr{T}_{n_{j}}\right\}_{j \geq 0}$ of $\left\{\mathscr{T}_{n}\right\}$ which satisfies $\mathscr{T}_{n_{j}}<\mathscr{T}_{n_{j}+1}$ for all $j \geq 0$. Also consider that the sequence of integers $\{\delta(n)\}_{n \geq n_{0}}$ is defined by

$$
\delta(n)=\max \left\{k \leq n \mid \mathscr{T}_{k}<\mathscr{T}_{k+1}\right\}
$$

Then $\{\delta(n)\}_{n \geq n_{0}}$ is a nondecreasing sequence verifying $\lim _{n \rightarrow \infty} \delta(n)=\infty, \forall n \geq n_{0}$; it holds that $\mathscr{T}_{\delta(n)}<\mathscr{T}_{\delta(n)+1}$ and one has

$$
\mathscr{T}_{n}<\mathscr{T}_{\delta(n)+1}
$$

Lemma 13. Let $K$ be a closed convex subset of a real Hilbert space $H$, given $x \in H$ and $y \in K$. Then $y=P_{K} x$ if and only if there holds the inequality

$$
\langle x-y, y-z\rangle \geq 0, \quad \forall z \in K \text {. }
$$

\section{Main Results}

Let $C$ be a nonempty closed convex subset of a real Hilbert space $H$, and let $T_{1}, T_{2}: C \rightarrow C$ be $k_{1}-, k_{2}$-strictly pseudononspreading mappings with nonempty fixed point set $\Omega=F_{i x}\left(T_{1}\right) \bigcap F_{i x}\left(T_{2}\right)$. Let $f$ be a $L$-Lipschitz mapping on $H$ with coefficient $L>0$. Assume that the set $\Omega$ is nonempty. Since $\Omega$ is closed and convex, the nearest point projection from $C$ onto $\Omega$ is well defined. Recall that $B: H \rightarrow H$ is $\eta$-strongly monotone and $k$-Lipschitzian on $H$ with $k>0$, $\eta>0$.

Lemma 14 (see [13]). Let $H$ be a real Hilbert space and let $B$ be a $k$-Lipschitzian and $\eta$-strongly monotone operator with $k>0$, $\eta>0$. Let $0<\mu<\left(2 \eta / k^{2}\right)$ and $\tau=\mu\left(\eta-\left(\eta k^{2} / 2\right)\right)$. Then for $t \in \min \{1,(1 / \tau)\}, I-t \mu B$ is in contradiction with constant $1-t \tau$.

Lemma 15. Let $T$ be a $k$-strictly pseudononspreading mapping on $H$, and $T_{\omega} x=(1-\omega) x+\omega T x$ with $0<k<\omega<1 / 2$. Then

$$
\begin{aligned}
& \left\|T_{\omega} x-T_{\omega} y\right\|^{2} \\
& \quad \leq\|x-y\|^{2}+\frac{2}{\omega}\left\langle x-T_{\omega} x, y-T_{\omega} y\right\rangle, \quad \forall x, y \in C .
\end{aligned}
$$

Proof. For $x, y \in H$, we have

$$
\begin{aligned}
\| T_{\omega} x- & T_{\omega} y \|^{2} \\
= & (1-\omega)\|x-y\|^{2}+\omega\|T x-T y\|^{2} \\
& -\omega(1-\omega)\|x-T x-(y-T y)\|^{2} \\
\leq & (1-\omega)\|x-y\|^{2} \\
& +\omega\left[\|x-y\|^{2}+k\|x-T x-(y-T y)\|^{2}\right. \\
& \quad+2\langle x-T x, y-T y\rangle] \\
& -\omega(1-\omega)\|x-T x-(y-T y)\|^{2} \\
= & \|x-y\|^{2}+2 \omega\langle x-T x, y-T y\rangle \\
& -\omega(1-\omega)(1-k)\|x-T x-(y-T y)\|^{2} \\
\leq & \|x-y\|^{2}+2 \omega\langle x-T x, y-T y\rangle \\
= & \|x-y\|^{2}+\frac{2}{\omega}\langle x-T \omega x, y-T \omega y .
\end{aligned}
$$

Remark 16. If $y=p \in F_{i x}(T)$ and from Lemma 15, we can easily claim that $\left\|T_{\omega} x-p\right\| \leq\|x-p\|, \forall x \in C$.

Theorem 17. Let $C$ be a nonempty, closed, and convex subset of a real Hilbert space $H$. Let $T_{1}, T_{2}: C \rightarrow C$ be $k_{1}-, k_{2^{-}}$ strictly pseudononspreading mappings and demiclosed. Let $f$ be L-Lipschitz mapping on $H$ with coefficient $L>0$ and let $B: H \rightarrow H$ be $\eta$-strongly monotone and $k$-Lipschitzian on $H$ with $k>0, \eta>0,0<k_{1}$, and $k_{2}<\omega<1 / 2$. Let the sequence of $\left\{\alpha_{n}\right\}$ and $\left\{\beta_{n}\right\}$ satisfy the following conditions:

$$
\begin{aligned}
& \text { (c1) } \lim _{n \rightarrow \infty} \alpha_{n}=0, \\
& \text { (c2) } \sum_{n=0}^{\infty} \alpha_{n}=\infty \text { and } \lim _{n \rightarrow \infty} \alpha_{n+1} / \alpha_{n}=1 \text {, } \\
& \text { (c3) } 0<c \leq \beta_{n} \leq d<1 .
\end{aligned}
$$

Let $0<\mu<2 \eta / k^{2}, 0<\gamma<\mu\left(\eta-\left(\mu k^{2} / 2\right)\right) / L=\tau / L$, and let the following sequence $\left\{x_{n}\right\}_{n=0}^{\infty}$ be a sequence in $C$ generated from an arbitrary $x_{1} \in C$ by

$$
\begin{aligned}
x_{n+1}= & \beta_{n} x_{n}+\left(1-\beta_{n}\right) \\
& \times T_{\omega_{1}}\left(\alpha_{n} \gamma f\left(x_{n}\right)+\left(I-\mu \alpha_{n} B\right) T_{\omega_{2}} x_{n}\right),
\end{aligned}
$$

where $T_{\omega_{i}}=\left(1-\omega_{i}\right) I+\omega_{i} T_{i}$ with $0<k_{i}<\omega_{i}<1, i=1,2$. Then $\left\{x_{n}\right\}$ converges strongly to the unique element $x^{*}$ in $\Omega=$ $F_{i x}\left(T_{1}\right) \bigcap F_{i x}\left(T_{2}\right)$ verifying

$$
x^{*}=\left(P_{\Omega} \cdot f\right) x^{*},
$$

which equivalently solves the following variational inequality problem:

$$
x^{*} \in \Omega, \quad\left\langle(\gamma f-\mu B) x^{*}, v-x^{*}\right\rangle \leq 0, \quad \forall v \in \Omega .
$$


Proof. Let $x^{*} \in \Omega$. According to Remark 16, we have

$$
\left\|T_{\omega} x_{n}-x^{*}\right\| \leq\left\|x_{n}-x^{*}\right\| .
$$

Let $y_{n}=\alpha_{n} \gamma f\left(x_{n}\right)+\left(I-\mu \alpha_{n} B\right) T_{\omega_{2}} x_{n}$. From (22), we obtain

$$
\begin{aligned}
\left\|x_{n+1}-x^{*}\right\| & =\left\|\beta_{n} x_{n}+\left(1-\beta_{n}\right) T_{\omega_{1}} y_{n}-x^{*}\right\| \\
& \leq \beta_{n}\left\|x_{n}-x^{*}\right\|+\left(1-\beta_{n}\right)\left\|T_{\omega_{1}} y_{n}-x^{*}\right\| \\
& \leq \beta_{n}\left\|x_{n}-x^{*}\right\|+\left(1-\beta_{n}\right)\left\|y_{n}-x^{*}\right\|,
\end{aligned}
$$

And, from Lemma 14, we also obtain that

$$
\begin{aligned}
\left\|y_{n}-x^{*}\right\|= & \| \alpha_{n} \gamma\left(f\left(x_{n}\right)-f\left(x^{*}\right)\right) \\
& +\left(I-\mu \alpha_{n} B\right)\left(T_{\omega_{2}} x_{n}-x^{*}\right) \\
& +\alpha_{n}\left(\gamma f\left(x^{*}\right)-\mu B x^{*}\right) \| \\
\leq & \alpha_{n} \gamma\left\|f\left(x_{n}\right)-f\left(x^{*}\right)\right\| \\
& +\left(1-\alpha_{n} \tau\right)\left\|T_{\omega_{2}} x_{n}-x^{*}\right\| \\
& +\alpha_{n}\left\|\gamma f\left(x^{*}\right)-\mu B x^{*}\right\| \\
\leq & \alpha_{n} \gamma L\left\|x_{n}-x^{*}\right\| \\
& +\left(1-\alpha_{n} \tau\right)\left\|x_{n}-x^{*}\right\| \\
& +\alpha_{n}\left\|\gamma f\left(x^{*}\right)-\mu B x^{*}\right\| .
\end{aligned}
$$

Together with (26) and (27), we obtain

$$
\begin{aligned}
\| x_{n+1}- & x^{*} \| \\
\leq & {\left[1-\alpha_{n}\left(1-\beta_{n}\right)(\tau-\gamma L)\right]\left\|x_{n}-x^{*}\right\| } \\
& +\alpha_{n}\left(1-\beta_{n}\right)\left\|\gamma f\left(x^{*}\right)-\mu B x^{*}\right\| .
\end{aligned}
$$

Putting $M_{1}=\max \left\{\left\|x_{0}-x^{*}\right\|,\left\|\gamma f\left(x^{*}\right)-x^{*}\right\|\right\}$, we clearly obtain $\left\|x_{n}-x^{*}\right\| \leq M_{1}$. By induction, we can deduce that $\left\{x_{n}\right\}$ is bounded and the sequences $\left\{f\left(x_{n}\right)\right\}$ and $\left\{T_{\omega_{i}} x_{n}\right\}, i=1,2$, are also bounded.

Next, we show the following estimation:

$$
\begin{aligned}
\mathscr{T}_{n+1} \leq & \left(1-\alpha_{n} \delta\right) \mathscr{T}_{n} \\
& -\delta\left(\left\|x_{n}-T_{2} x_{n}\right\|^{2}+\left\|T_{\omega_{1}} y_{n}-x_{n}\right\|^{2}\right) \\
& +2 \alpha_{n}\left(1-\beta_{n}\right)\left\langle\gamma f\left(x_{n}\right)-\mu B x^{*}, y_{n}-x^{*}\right\rangle,
\end{aligned}
$$

where $\mathscr{T}_{n}=\left\|x_{n}-x^{*}\right\|^{2}$ and $\delta>0$ is chosen so that

$$
\begin{gathered}
\delta \leq \min \left\{\omega_{2}\left(1-\alpha_{n} \tau\right)\left(1-\beta_{n}\right)\left(1-\omega_{2}\right)\left(1-k_{2}\right),\right. \\
\left.\beta_{n}\left(1-\beta_{n}\right)\right\} .
\end{gathered}
$$

From Lemma 15, we obtain

$$
\begin{aligned}
& \left\|T_{\omega_{2}} x_{n}-x^{*}\right\|^{2} \\
& \quad \leq\left\|x_{n}-x^{*}\right\|^{2}-\omega_{2}\left(1-\omega_{2}\right)\left(1-k_{2}\right)\left\|x_{n}-T_{2} x_{n}\right\|^{2} .
\end{aligned}
$$

By (25) and (31), we get

$$
\begin{aligned}
\| y_{n}- & x^{*} \|^{2} \\
= & \left\|\alpha_{n}\left(\gamma f\left(x_{n}\right)-\mu B x^{*}\right)+\left(I-\mu \alpha_{n} B\right)\left(T_{\omega_{2}} x_{n}-x^{*}\right)\right\|^{2} \\
\leq & \left(1-\alpha_{n} \tau\right)\left\|T_{\omega_{2}} x_{n}-x^{*}\right\|^{2} \\
& +2 \alpha_{n}\left\langle\gamma f\left(x_{n}\right)-\mu B x^{*}, y_{n}-x^{*}\right\rangle \\
\leq & \left(1-\alpha_{n} \tau\right)\left\|x_{n}-x^{*}\right\|^{2} \\
& -\omega_{2}\left(1-\alpha_{n} \tau\right)\left(1-\omega_{2}\right)\left(1-k_{2}\right)\left\|x_{n}-T_{2} x_{n}\right\|^{2} \\
& +2 \alpha_{n}\left\langle\gamma f\left(x_{n}\right)-\mu B x^{*}, y_{n}-x^{*}\right\rangle .
\end{aligned}
$$

Consequently,

$$
\begin{aligned}
\| x_{n+1}- & x^{*} \|^{2} \\
= & \beta_{n}\left\|x_{n}-x^{*}\right\|^{2}+\left(1-\beta_{n}\right)\left\|T_{\omega_{1}} y_{n}-x^{*}\right\|^{2} \\
& -\beta_{n}\left(1-\beta_{n}\right)\left\|T_{\omega_{1}} y_{n}-x_{n}\right\|^{2} \\
\leq & \beta_{n}\left\|x_{n}-x^{*}\right\|^{2}-\beta_{n}\left(1-\beta_{n}\right)\left\|T_{\omega_{1}} y_{n}-x_{n}\right\|^{2} \\
& +\left(1-\beta_{n}\right)\left\|y_{n}-x^{*}\right\|^{2} \\
\leq & \beta_{n}\left\|x_{n}-x^{*}\right\|^{2}-\beta_{n}\left(1-\beta_{n}\right)\left\|T_{\omega_{1}} y_{n}-x_{n}\right\|^{2} \\
& +\left(1-\beta_{n}\right)\left(1-\alpha_{n} \tau\right)\left\|x_{n}-x^{*}\right\|^{2} \\
& -\omega_{2}\left(1-\alpha_{n} \tau\right)\left(1-\beta_{n}\right)\left(1-\omega_{2}\right)\left(1-k_{2}\right) \\
& \times\left\|x_{n}-T_{2} x_{n}\right\|^{2} \\
& +2 \alpha_{n}\left(1-\beta_{n}\right)\left\langle\gamma f\left(x_{n}\right)-\mu B x^{*}, y_{n}-x^{*}\right\rangle \\
\leq & \left(1-\alpha_{n} \tau\left(1-\beta_{n}\right)\right)\left\|x_{n}-x^{*}\right\|^{2} \\
& -\beta_{n}\left(1-\beta_{n}\right)\left\|T_{\omega_{1}} y_{n}-x_{n}\right\|^{2} \\
& -\omega_{2}\left(1-\alpha_{n} \tau\right)\left(1-\beta_{n}\right)\left(1-\omega_{2}\right)\left(1-k_{2}\right) \\
& \times\left\|x_{n}-T_{2} x_{n}\right\|^{2} \\
& 2 \alpha_{n}\left(1-\beta_{n}\right)\left\langle\gamma f\left(x_{n}\right)-\mu B x^{*}, y_{n}-x^{*}\right\rangle,
\end{aligned}
$$

and the desired inequality (29) follows.

Finally, we show $\lim _{n \rightarrow \infty} \mathscr{T}_{n}=0$ by considering two possible cases.

Case 1. $\left\{\mathscr{T}_{n}\right\}$ is nonincreasing sequence with some $n \geq$ $n_{0}$. In this case, $\left\{\mathscr{T}_{n}\right\}$ is then convergent because it is also nonnegative (hence it is bounded form blow). From (29), we have

$$
\delta\left(\left\|x_{n}-T_{2} x_{n}\right\|^{2}+\left\|T_{\omega_{1}} y_{n}-x_{n}\right\|^{2}\right) \leq \alpha_{n} M+\left(\mathscr{T}_{n}-\mathscr{T}_{n+1}\right),
$$


where $0<M=2\left\langle\gamma f\left(x_{n}\right)-\mu B x^{*}, y_{n}-x^{*}\right\rangle$. Consequently, both $\left\|x_{n}-T_{2} x_{n}\right\|$ and $\left\|T_{\omega_{1}} y_{n}-x_{n}\right\|$ converge to zero. From (22) and (25), $\lim _{n \rightarrow \infty} \alpha_{n}=0$ and the sequence $\left\{x_{n}\right\}$ is bounded, we obtain

$$
\begin{aligned}
& \left\|y_{n}-x_{n}\right\| \\
& \quad=\left\|\alpha_{n}\left(\gamma f\left(x_{n}\right)-\mu B x_{n}\right)+\left(I-\mu \alpha_{n} B\right)\left(T_{\omega_{2}} x_{n}-x_{n}\right)\right\| \\
& \quad \leq \alpha_{n}\left\|\gamma f\left(x_{n}\right)-\mu B x_{n}\right\|+\left(1-\alpha_{n} \tau\right)\left\|T_{\omega_{2}} x_{n}-x_{n}\right\| \\
& \quad \leq \alpha_{n}\left\|\gamma f\left(x_{n}\right)-\mu B x_{n}\right\|+\left(1-\alpha_{n} \tau\right) \omega_{2}\left\|T_{2} x_{n}-x_{n}\right\| \longrightarrow 0,
\end{aligned}
$$

which implies

$$
\begin{aligned}
\left\|x_{n}-T_{\omega_{1}} x_{n}\right\|^{2} \leq & \left\|x_{n}-T_{\omega_{1}} y_{n}\right\|^{2}+\left\|T_{\omega_{1}} y_{n}-T_{\omega_{1}} x_{n}\right\|^{2} \\
\leq & \left\|x_{n}-T_{\omega_{1}} y_{n}\right\|^{2}+\left\|x_{n}-y_{n}\right\|^{2} \\
& +\frac{2}{\omega_{1}}\left\langle x_{n}-T_{\omega_{1}} x_{n}, y_{n}-T_{\omega_{1}} y_{n}\right\rangle \\
\leq & \left\|x_{n}-T_{\omega_{1}} y_{n}\right\|^{2}+\left\|x_{n}-y_{n}\right\|^{2} \\
& +\frac{2}{\omega_{1}}\left\langle x_{n}-T_{\omega_{1}} x_{n}, y_{n}-x_{n}\right\rangle \\
& +\frac{2}{\omega_{1}}\left\langle x_{n}-T_{\omega_{1}} x_{n}, x_{n}-T_{\omega_{1}} y_{n}\right\rangle .
\end{aligned}
$$

From $\lim _{n \rightarrow \infty}\left\|T_{\omega_{1}} y_{n}-x_{n}\right\|=0, \lim _{n \rightarrow \infty}\left\|y_{n}-x_{n}\right\|=0$, and $x_{n}-T_{\omega_{1}} x_{n}=\omega_{1}\left(x_{n}-T_{1} x_{n}\right)$, we obtain

$$
\lim _{n \rightarrow \infty}\left\|x_{n}-T_{1} x_{n}\right\|=\lim _{n \rightarrow \infty}\left\|x_{n}-T_{\omega_{1}} x_{n}\right\|=0 .
$$

Using the demiclosedness principle (Proposition 8) and (35), we know that $\omega_{w}\left(x_{n}\right) \in \Omega$; hence

$$
\begin{aligned}
\limsup _{n \rightarrow \infty}\left\langle\gamma f\left(x_{n}\right)-\mu B x^{*}, y_{n}-x^{*}\right\rangle \\
\quad=\limsup _{n \rightarrow \infty}\left\langle\gamma f\left(x_{n}\right)-\mu B x^{*}, x_{n}-x^{*}\right\rangle \\
=\max _{w \in \omega_{w}\left(x_{n}\right)}\left\langle\gamma f(w)-\mu B x^{*}, w-x^{*}\right\rangle \leq 0 .
\end{aligned}
$$

It then follows from (29) that

$$
\begin{aligned}
\mathscr{T}_{n+1} \leq & \left(1-\alpha_{n} \delta\right) \mathscr{T}_{n} \\
& +2 \alpha_{n}\left(1-\beta_{n}\right)\left\langle\gamma f\left(x_{n}\right)-\mu B x^{*}, y_{n}-x^{*}\right\rangle .
\end{aligned}
$$

According to Lemma 11, we obtain $\lim _{n \rightarrow \infty} \mathscr{T}_{n}=0$.

Case 2. Suppose there exists a subsequence $\left\{\mathscr{T}_{n_{k}}\right\}_{k \geq 0}$ of $\left\{\mathscr{T}_{n}\right\}_{n \geq 0}$ such that $\mathscr{T}_{n_{k}} \leq \mathscr{T}_{n_{k+1}}$ for all $k \geq 0$. In this situation, we consider the sequence of indices $\{\delta(n)\}$ as defined in Lemma 12. It follows that $\mathscr{T}_{\delta(n+1)}-\mathscr{T}_{\delta(n)}>0$, which by (29) amounts to

$$
\delta\left(\left\|x_{\delta(n)}-T_{2} x_{\delta(n)}\right\|^{2}+\left\|T_{\omega_{1}} y_{\delta(n)}-x_{\delta(n)}\right\|^{2}\right) \leq \alpha_{\delta(n)} M .
$$

In a similar way to Case 1 , we obtain

$$
\limsup _{n \rightarrow \infty}\left\langle\gamma f\left(x_{\delta(n)}\right)-\mu B x^{*}, y_{\delta(n)}-x^{*}\right\rangle \leq 0 .
$$

From Lemma 12 and (29)

$$
\mathscr{T}_{\delta(n)} \leq 2\left(1-\beta_{\delta(n)}\right)\left\langle\gamma f\left(x_{\delta(n)}\right)-\mu B x^{*}, y_{\delta(n)}-x^{*}\right\rangle,
$$

for all $n>n_{0}$. Taking limsup in this inequality, we obtain $\lim _{n \rightarrow \infty} \mathscr{T}_{\delta(n)}=0$. Moreover, it follows from (22) that

$$
\begin{aligned}
\mathscr{T}_{\delta(n)+1}^{2} & =\left\|\left(x_{\delta(n)}-x^{*}\right)-\left(x_{\delta(n)}-x_{\delta(n)+1}\right)\right\|^{2} \\
& \leq \mathscr{T}_{\delta(n)}^{2}+\left\|x_{\delta(n)}-x_{\delta(n)+1}\right\|^{2} \\
& \leq \mathscr{T}_{\delta(n)}^{2}+\left\|x_{\delta(n)}-T_{\omega_{1}} y_{\delta(n)}\right\|^{2},
\end{aligned}
$$

which together with (40) implies $\lim _{n \rightarrow \infty} \mathscr{T}_{\delta(n)+1}=0$. Consequently, from Lemma 12, we obtain $\lim _{n \rightarrow \infty} \mathscr{T}_{n}=0$.

In addition, from (38), we obtain

$$
\begin{aligned}
& \limsup _{n \rightarrow \infty}\left\langle\gamma f\left(x_{n}\right)-\mu B x^{*}, x_{n}-x^{*}\right\rangle \\
& =\limsup _{n \rightarrow \infty}\left\langle\gamma f\left(x_{n}\right)-\gamma f\left(x^{*}\right), x_{n}-x^{*}\right\rangle \\
& \quad+\limsup _{n \rightarrow \infty}\left\langle\gamma f\left(x^{*}\right)-\mu B x^{*}, x_{n}-x^{*}\right\rangle \\
& =\gamma L \lim _{n \rightarrow \infty} \mathscr{T}_{n}+\limsup _{n \rightarrow \infty}\left\langle\gamma f\left(x^{*}\right)-\mu B x^{*}, x_{n}-x^{*}\right\rangle \\
& =\left\langle\gamma f\left(x^{*}\right)-\mu B x^{*}, \bar{y}-x^{*}\right\rangle, \quad \bar{y} \in \Omega ;
\end{aligned}
$$

then variational inequality (44) can be written as

$$
\left\langle(I-\mu B+\gamma f) x^{*}-x^{*}, \bar{y}-x^{*}\right\rangle \geq 0, \quad \bar{y} \in \Omega .
$$

So, by Lemma 13, it is equivalent to the fixed point equation

$$
P_{F_{i x}(T)}(I-\mu B+\gamma f) x^{*}=x^{*} .
$$

Remark 18. For a nonspreading mapping $T$, we have $\varrho=0$ in Theorem 17 to obtain the following corollary.

Corollary 19. Let $C$ be a nonempty, closed, and convex subset of a real Hilbert space $H$. Let $T_{1}, T_{2}: H \rightarrow H$ be nonspreading mappings and demiclosed. Let $f$ be L-Lipschitz mapping on $H$ with coefficient $L>0$ and let $B: H \rightarrow H$ be $\eta$-strongly monotone and $k$-Lipschitzian on $H$ with $k>0, \eta>0$. Let the sequence of $\left\{\alpha_{n}\right\}$ and $\left\{\beta_{n}\right\}$ satisfy the following conditions:

(c1) $\lim _{n \rightarrow \infty} \alpha_{n}=0$,

(c2) $\sum_{n=0}^{\infty} \alpha_{n}=\infty$ and $\lim _{n \rightarrow \infty} \alpha_{n+1} / \alpha_{n}=1$,

(c3) $0<c \leq \beta_{n} \leq d<1$.

Let the following sequence $\left\{x_{n}\right\}_{n=0}^{\infty}$ be a sequence in $C$ generated from an arbitrary $x_{1} \in C$ by

$$
x_{n+1}=\beta_{n} x_{n}+\left(1-\beta_{n}\right) T_{\omega_{1}}\left(\alpha_{n} \gamma f\left(x_{n}\right)+\left(I-\mu \alpha_{n} B\right) T_{\omega_{2}} x_{n}\right) \text {, }
$$


where $T_{\omega_{i}}=\left(1-\omega_{i}\right) I+\omega_{i} T_{i}, \omega_{i} \in(0,1), i=1,2$. Then $\left\{x_{n}\right\}$ converges strongly to the unique element $x^{*}$ in $\Omega=$ $F_{i x}\left(T_{1}\right) \bigcap F_{i x}\left(T_{2}\right)$ verifying

$$
x^{*}=\left(P_{\Omega} \cdot f\right) x^{*},
$$

which equivalently solves the following variational inequality problem:

$$
x^{*} \in \Omega \quad\left\langle(\gamma f-\mu B) x^{*}, v-x^{*}\right\rangle \leq 0, \quad \forall v \in \Omega .
$$

Remark 20. If $B=I$ and $\mu=1$ in Corollary 19, we obtain the following corollary.

Corollary 21. Let $C$ be a nonempty, closed, and convex subset of a real Hilbert space $H$. Let $T_{1}, T_{2}: H \rightarrow H$ be nonspreading mappings and demiclosed. Let $f$ be L-Lipschitz mapping on $H$ with coefficient $L>0$. Let the sequence of $\left\{\alpha_{n}\right\}$ and $\left\{\beta_{n}\right\}$ satisfy the following conditions:

(c1) $\lim _{n \rightarrow \infty} \alpha_{n}=0$,

(c2) $\sum_{n=0}^{\infty} \alpha_{n}=\infty$ and $\lim _{n \rightarrow \infty} \alpha_{n+1} / \alpha_{n}=1$,

(c3) $0<c \leq \beta_{n} \leq d<1$.

Let the following sequence $\left\{x_{n}\right\}_{n=0}^{\infty}$ be a sequence in $C$ generated from an arbitrary $x_{1} \in C$ by

$$
x_{n+1}=\beta_{n} x_{n}+\left(1-\beta_{n}\right) T_{\omega_{1}}\left(\alpha_{n} \gamma f\left(x_{n}\right)+\left(1-\alpha_{n}\right) T_{\omega_{2}} x_{n}\right),
$$

where $T_{\omega_{i}}=\left(1-\omega_{i}\right) I+\omega_{i} T_{i}, \omega_{i} \in(0,1), i=1,2$. Then $\left\{x_{n}\right\}$ converges strongly to the unique element $x^{*}$ in $\Omega=$ $F_{i x}\left(T_{1}\right) \bigcap F_{i x}\left(T_{2}\right)$ verifying

$$
x^{*}=\left(P_{\Omega} \cdot f\right) x^{*},
$$

which equivalently solves the following variational inequality problem:

$$
x^{*} \in \Omega, \quad\left\langle(\gamma f-I) x^{*}, v-x^{*}\right\rangle \leq 0, \quad \forall v \in \Omega .
$$

\section{Application}

In this section, we constructed a numerical example to illustrate that our main results are well defined. The following example is introduced by Deng et al. [14]. They prove that $T$ is $(1 / 3)$-strictly pseudononspreading.

Example 22. Let $X=l^{2}$ with the norm $\|\cdot\|$ defined by

$$
\|x\|=\sqrt{\sum_{i=1}^{\infty} x_{i}^{2}}, \quad \forall x=\left(x_{1}, x_{2}, \ldots, x_{n}, \ldots\right) \in X,
$$

$C=\left\{x=\left(x_{1}, x_{2}, \ldots, x_{n}, \ldots\right) \mid x_{i} \in R^{1}, i=1,2, \ldots\right\}$, and let $C$ be an orthogonal subspace of $X$ (i.e., $\forall x, y \in C$, we have $\langle x, y\rangle=0)$. Then it is obvious that $C$ is a nonempty closed convex subset of $X$. Now, for any $x=\left(x_{1}, x_{2}, \ldots, x_{n}, \ldots\right) \in C$, define a mapping $T: C \rightarrow C$ as follows:

$$
T x= \begin{cases}\left(x_{1}, x_{2}, \ldots, x_{n}, \ldots\right), & \prod_{i=1}^{\infty} x_{i}<0, \\ \left(-2 x_{1},-2 x_{2}, \ldots,-2 x_{n}, \ldots\right), & \prod_{i=1}^{\infty} x_{i} \geq 0 .\end{cases}
$$

From the definition of this example, let $x=\left(x_{1}, x_{2}\right) \in R^{2}$ and $f(x)=8\left(\sin \left(x_{1}\right), \cos \left(x_{2}\right)\right)$ be a Lipschitz mapping on $R^{2}$. Assume that $T_{1}(x)=\left(0,4 x_{1}\right)$ and $T_{2}(x)=\left(4 x_{2}, 0\right)$ be $(1 / 3)$ strictly pseudononspreading mappings. Let $\alpha_{n}=\beta_{n}=1 / 5$, $\gamma=1 / 8, \mu=\omega_{1}=\omega_{2}=1$, and $B=I$. According to (22), we can obtain the following algorithm:

$$
x_{n+1}=\frac{1}{5} x_{n}+\frac{4}{200} T_{1}\left(f\left(x_{n}\right)\right)+\frac{16}{25} T_{1}\left(T_{2}\left(x_{n}\right)\right) .
$$

From Theorem 17, we can easily know that algorithm (55) converges to the unique point in $R^{2}$. Let $x_{0}=(5,4)$ and $\left|x_{n+1}-x_{n}\right| \leq 10^{-5}$, and let $x^{*}$ be the fixed point of the algorithm (55). Using the software of MATLAB, we obtain $x^{*}=(0,0)$.

\section{Acknowledgments}

This work is supported in part by National Natural Science Foundation of China (71272148), the Ph.D. Programs Foundation of Ministry of Education of China (20120032110039), and China Postdoctoral Science Foundation (Grant no. 20100470783).

\section{References}

[1] S. Plubtieng and R. Punpaeng, "A new iterative method for equilibrium problems and fixed point problems of nonexpansive mappings and monotone mappings," Applied Mathematics and Computation, vol. 197, no. 2, pp. 548-558, 2008.

[2] L. C. Zeng, S. Schaible, and J. C. Yao, "Iterative algorithm for generalized set-valued strongly nonlinear mixed variationallike inequalities," Journal of Optimization Theory and Applications, vol. 124, no. 3, pp. 725-738, 2005.

[3] M. O. Osilike and F. O. Isiogugu, "Weak and strong convergence theorems for nonspreading-type mappings in Hilbert spaces," Nonlinear Analysis: Theory, Methods \& Applications, vol. 74, no. 5, pp. 1814-1822, 2011.

[4] W. R. Mann, "Mean value methods in iteration," Proceedings of the American Mathematical Society, vol. 4, pp. 506-510, 1953.

[5] W. Takahashi and T. Tamura, "Convergence theorems for a pair of nonexpansive mappings," Journal of Convex Analysis, vol. 5, no. 1, pp. 45-56, 1998.

[6] M. Tian, "A general iterative algorithm for nonexpansive mappings in Hilbert spaces," Nonlinear Analysis: Theory, Methods \& Applications, vol. 73, no. 3, pp. 689-694, 2010.

[7] T. L. Hicks and J. D. Kubicek, "On the Mann iteration process in a Hilbert space," Journal of Mathematical Analysis and Applications, vol. 59, no. 3, pp. 498-504, 1977.

[8] S. A. Naimpally and K. L. Singh, "Extensions of some fixed point theorems of Rhoades," Journal of Mathematical Analysis and Applications, vol. 96, no. 2, pp. 437-446, 1983.

[9] P. E. Maing, "The viscosity approximation process for quasinonexpansive mappings in Hilbert spaces," Computers \& Mathematics with Applications, vol. 59, no. 1, pp. 74-79, 2010.

[10] N. Petrot and R. Wangkeeree, "A general iterative scheme for strict pseudononspreading mapping related to optimization problem in Hilbert spaces," Journal of Nonlinear Analysis and Optimization, vol. 2, no. 2, pp. 329-336, 2011. 
[11] H. K. Xu, "Iterative algorithms for nonlinear operators," Journal of the London Mathematical Society, vol. 66, no. 1, pp. 240-256, 2002.

[12] P. E. Maing, "Strong convergence of projected subgradient methods for nonsmooth and nonstrictly convex minimization," Set-Valued Analysis, vol. 16, no. 7-8, pp. 899-912, 2008.

[13] H. Piri, "A general iterative method for finding common solutions of system of equilibrium problems, system of variational inequalities and fixed point problems," Mathematical and Computer Modelling, vol. 55, no. 3-4, pp. 1622-1638, 2012.

[14] B. C. Deng, T. Chen, and Z. F. Li, "Viscosity iteration algorithm for a $\varrho$-strictly pseudononspreading mapping in a Hilbert space," Journal of Inequalities and Applications, vol. 2013, article 80, 2013. 


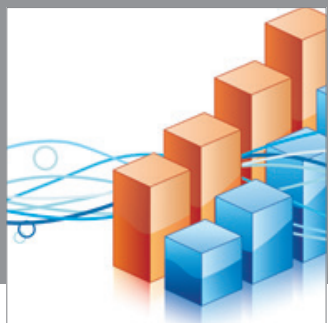

Advances in

Operations Research

mansans

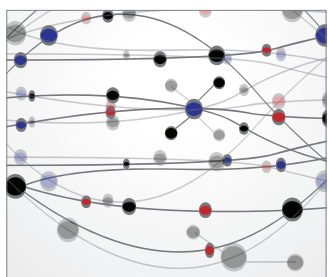

The Scientific World Journal
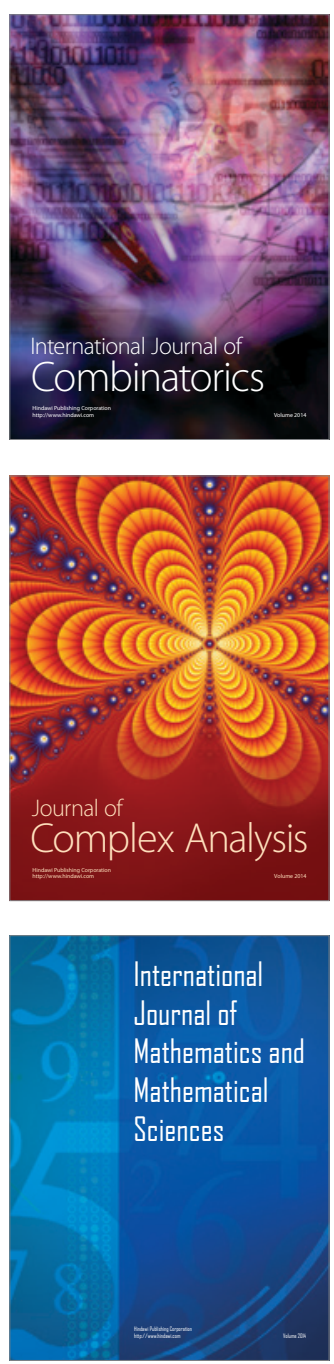
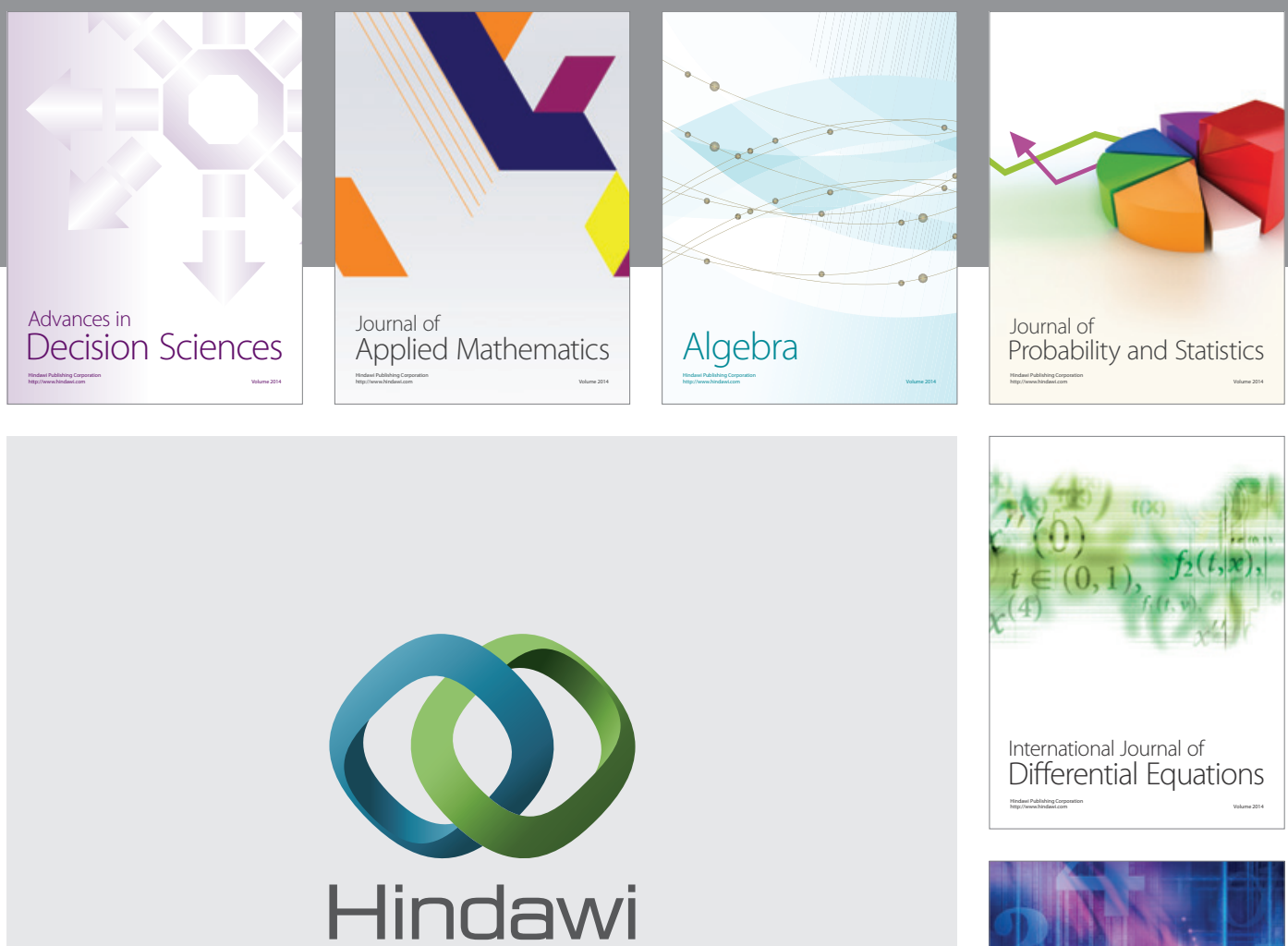

Submit your manuscripts at http://www.hindawi.com
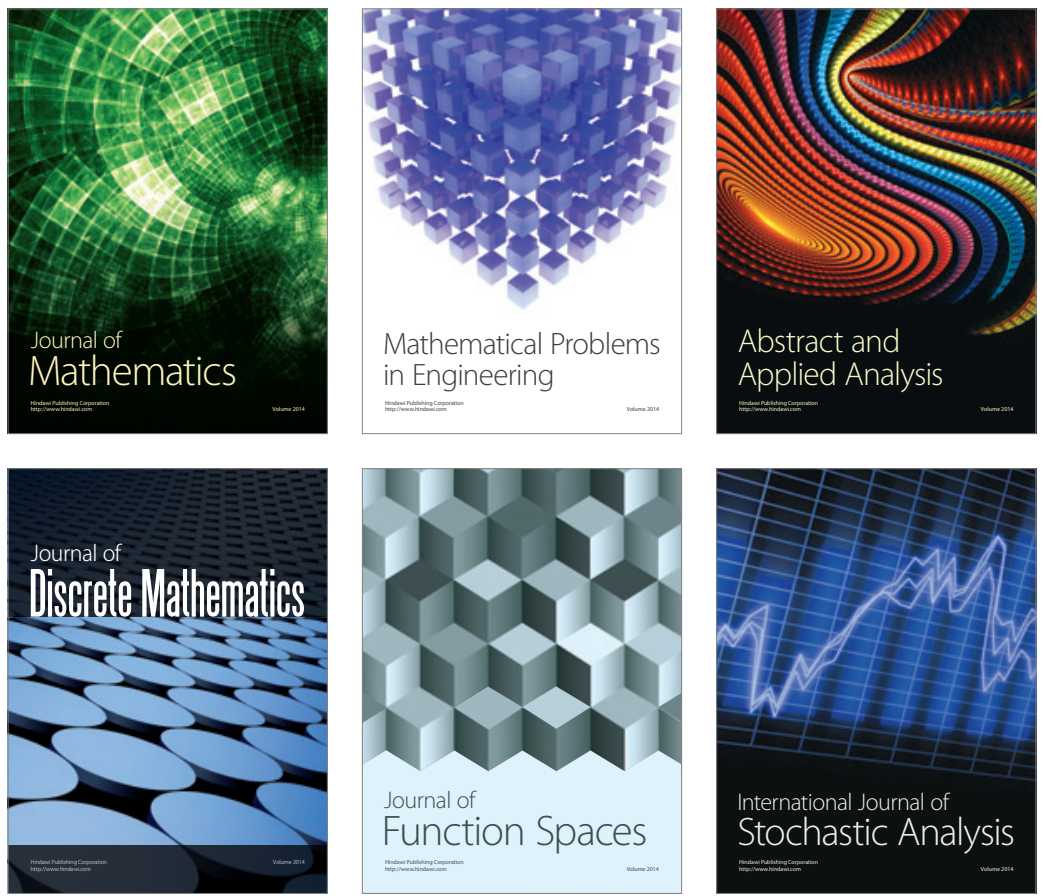

Journal of

Function Spaces

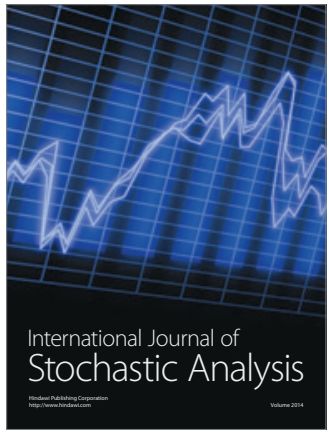

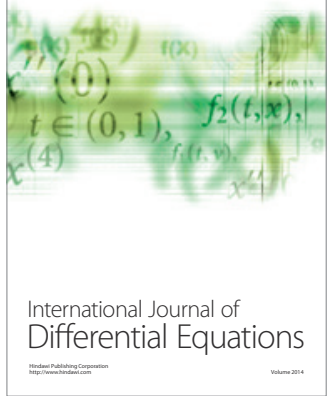
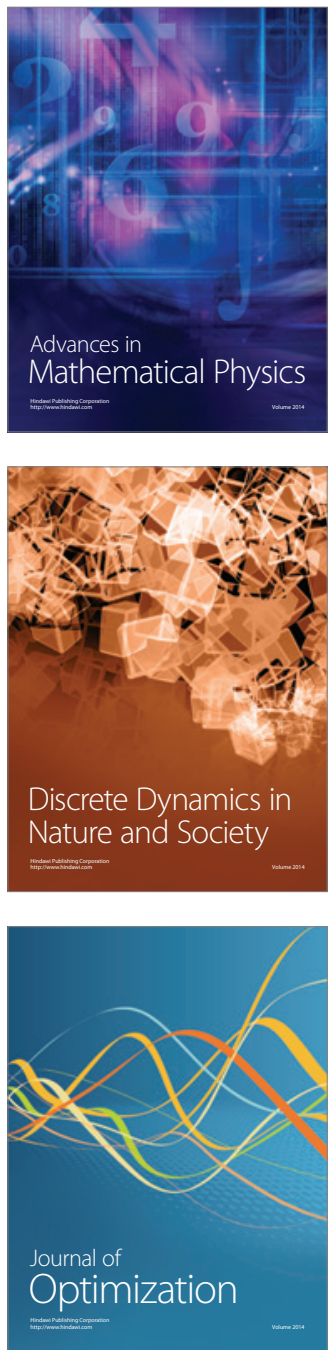